\title{
Francisolā
}

\section{Congrès CAP-Kyoto2017 : la place de la diversité des langues en didactique du français}

\author{
Jonathan GOUJON
}

Département Global Communication, Université Doshisha - Japon

Reçu le 20 avril 2018 | Évalué le 25 avril 2018 | Accepté le 18 juin 2018

\begin{abstract}
RÉSUMÉ. Vingt ans après la signature du protocole de Kyoto de 1997, le $4^{\text {ème }}$ congrès régional de la Commission Asie-Pacifique de la Fédération Internationale des Professeurs de Français s'est tenu à Kyoto même, afin de promouvoir le français dans un souci de préserver l'environnement linguistique, voire de faire de celui-ci, via la francophonie, un garant de la protection de la diversité des langues et des cultures. Bien que l'intention soit louable et sa franchise indiscutable, il est difficile de cerner concrètement les pourtours de cette démarche. Nous présentons dans cet article une recherche basée sur une enquête post-congrès et sur l'analyse du programme dudit congrès pour saisir des représentations que les enseignants-chercheurs en didactique du français se font de cette diversité. Les résultats de ces analyses font comprendre que si la francophonie est réellement vécue, elle et la diversité des langues sont insuffisamment exprimées.
\end{abstract}

Mots-clés : didactique des langues, diversité des langues et des cultures, francophone, francophonophile, langues francophones

\begin{abstract}
Twenty years after Kyoto Protocol was adopted in 1997, the $4^{\text {th }}$ regional convention of the Asia-Pacific Committee belonging to the International Federation of the French Language Teachers took place in Kyoto to promote the French language in the current climate of language preservation. The tendency is to make the French language a protector of linguistic and cultural diversity. However, this approach is hard to define, regardless the genuine intentions. Based on a post-convention survey of the attendees and on an analysis of the convention's programme, this research helps to understand some representations that university lecturers and researchers of the French language education have about this diversity. The results of those analysis show that francophonie (the French-speaking world) and linguistic diversity are not much expressed, even though francophonie is well experienced.
\end{abstract}

Keywords : diversity of languages and cultures, francophone, francophonophile, French-speaking languages, Language education

auteur correspondant : goujonathan.fr@gmail.com

Pour citer cet article (Style APA) : Goujon, J. (2018). Congrès CAP-Kyoto2017 : la place de la diversité des langues en didactique du français. Francisola: Revue Indonésienne de la langue et la littérature françaises, 3(1), 54-62. doi: 10.17509/francisola.v3i1.11891 


\section{INTRODUCTION}

A l'heure où les besoins d'un développement durable des langues vont grandissant, la Fédération Internationale des Professeurs de Français (dorénavant FIPF) et ses membres se sont retrouvés à Kyoto du 20 au 24 septembre 2017 pour partager leurs réflexions sur l'écologie du français et la diversité des langues. Organisé par la Société Japonaise de Didactique du Français (SJDF), ce 4ème congrès de la Commission AsiePacifique (CAP-Kyoto2017), forte de ses 24 organisations issues de 22 pays différents, a $\mathrm{pu}$ proposer des interventions sur 7 axes thématiques allant de la protection de la diversité culturelle et linguistique à la formation des enseignants. Ainsi plus de 200 communications, une trentaine de tables rondes, une dizaine d'ateliers et de conférences ont été dispensées aussi bien par des didacticiens de FLE invités, dont le degré d'expertise n'est plus à prouver, que par des écrivains francophones de renommée internationale, ou des professionnels de l'enseignement venus de 56 pays, faisant de CAP-Kyoto2017, le congrès le plus conséquent qu'il y ait eu jusqu'à présent (Komatsu, 2017a). Il fut donc une formidable occasion d'échanger sur les pratiques pédagogiques, les points de vue didactiques et surtout sur les chemins que doit prendre l'enseignement de la langue française dans une ère où la mondialisation et l'uniformisation menacent la diversité. Pour Klinkenberg (2015), une des premières missions de la francophonie est de se présenter comme garante de la diversité et des cultures dont leur réduction constitue une menace réelle. En ce sens, trois défis sont, pour lui, à relever: le premier est de combattre la centralisation, le deuxième est de modifier le discours sur la langue française en changeant son image élitiste, celle d'une langue difficile, sans renier l'image d'une langue raffinée. Enfin, le troisième défi est celui de s'ouvrir au monde extérieur. La langue française et son enseignement ont un rôle important à jouer dans la préservation des langues, de par leur omniprésence sur notre planète. Rappelons, comme le soulève l'Observatoire de la langue française de l'Organisation Internationale de la Francophonie (désormais OIF), qu'elle est avec l'anglais la seule langue enseignée dans le monde entier. (Observatoire de la langue française de l'OIF, 2014, p. 12).

Si le français a un rôle à jouer dans l'écologie des langues, il faut, pour se faire, cerner son biotope et ses biocénoses dans le cadre de notre discipline, celle de la didactique du français. Ce travail vise ainsi à saisir les représentations que des acteurs primordiaux en didactique du français, les enseignants-chercheurs, se font de la francophonie et de la diversité des langues. Simultanément, nous nous efforçons de définir une terminologie à employer et des pistes à emprunter.

\section{MÉTHODE}

Cette recherche repose en grande partie sur une enquête menée un mois après le congrès auprès des congressistes et sur le programme publié des interventions (SJDF, 2017). L'enquête interroge les participants sur le déroulement du congrès, la logistique, leur satisfaction, mais aussi sur le contenu des interventions, leurs représentations de la langue enseignée, de ce qu'est la francophonie et la diversité des langues dans notre discipline. Des 400 congressistes, 120 personnes ont répondu favorablement. L'enquête est constituée de 6 parties. La première cerne le profil des répondants, avec 8 questions sur les formations pédagogiques suivies, le pays d'enseignement, ou les matières enseignées. La deuxième partie porte sur l'organisation même du congrès, la logistique et demande aux répondants leur degré de satisfaction en 14 questions. Sont éludées, dans ce travail, les questions relatives à l'organisation car elles ne nous apportent pas d'informations utiles au développement de la didactique des langues. La troisième partie aborde les activités culturelles et leur réception en 14 questions. Les langues utilisées lors du congrès et leurs 13 questions constituent la quatrième partie. La cinquième partie a pour objet les interventions telles que les communications, les ateliers et conférences en 23 questions; quand la sixième partie implique des appréciations plus générales en 2 questions. Cette enquête, assez conséquente, compte au 
total 74 questions; généralement à choix multiples (54), mais aussi semi-directives (20), offrant la possibilité de préciser certains points évoqués. Le fait qu'un quart des congressistes a répondu émet des réserves quant à la représentativité des enseignantschercheurs présents au congrès. Néanmoins, les résultats de cette enquête donnent des pistes sur la vision, partagée ou non, de ce qu'est la diversité des langues et la francophonie; tant ce qui nous intéresse ici n'est pas le résultat dans l'absolu mais en relatif.

Le programme du congrès, quant-àlui, met en valeur le vocabulaire employé par les intervenants, reflétant les différentes conceptions des thèmes abordés et objectivant les liens ou les disjonctions entre les réponses des enquêtes et les présentations des communications. Les lettres adressées aux participants en début de programme par les différents représentants des institutions officielles n'ont pas été prises en compte. Celles-ci, ayant été spécifiquement rédigées pour l'évènement, intègrent des termes fédérateurs, généraux, partagés par tous.

\section{RÉSULTATS ET DISCUSSION}

De ce travail, il est à constater que des notions majeures du congrès renvoyaient à différentes significations, illustrant ici même la diversité étudiée, mais faisant naître, par la même occasion, des ambiguïtés entravant la réflexion didactique malgré des préoccupations communes.

\subsection{Le Francophone en didactique des langues}

Deux exemples frappants sont les substantifs «francophonie» et «francophone». Il est à se demander s'ils sont au service de la diversité qu'ils sont censés défendre.

Si la distinction entre Francophonie institutionnelle (Awad et Thibeault, 2017 ; Traisnel, 2015) et francophonie, en tant que communauté humaine (Traisnel, 2015), est aujourd'hui très claire, le terme "francophonie » cherche encore à se définir. Il a été, tout d'abord, délicat à employer de par son renvoi à une histoire douloureuse. Senghor utilisait en 1960 à terme égal francophonie et francité. Pour Jacques Godbout, en évoquant la laïcité, le mot francité recouvrait partiellement celui de consensus, quand le terme francophonie « rimait avec colonie » (Berthelot, 2015, p. 2628). Ce dernier terme suscitait également une litigieuse comparaison en renvoyant à une dévalorisation de certains utilisateurs de la langue française (Bonn, 2008, p. 43). La définition de francophonie apparaît floue dès 1962 dans la revue Esprit (Berthelot, 2015, p. 26). Elle correspondait, déjà à l'époque, aux communautés ayant le français comme langue maternelle, langue seconde ou langue privilégiée. Bien que le lien étroit de l'individu au français soit indéniable, les formes que prend la langue française sont moins visibles. De ce fait, Berthelot (2015, p. 25) dira : « le mot francophonie est un motvalise qui recouvre depuis sa création en 1880 par Onésime Reclus des réalités bien différentes qui peuvent être abordées selon plusieurs perspectives : linguistiques, historiques, didactiques, etc. »

En didactique du français, la signification du terme «francophone» semble desservir la diversité. Un francophone est « une personne capable de parler en français, quel que soit son niveau ou sa maîtrise d'autres compétences, comme l'écriture ou la lecture » (Observatoire de la langue française de l'OIF, 2014, p. 7). D'un point de vue de l'enseignement, nous ne savons pas à qui nous avons affaire, puisque les aptitudes et les expériences des apprenants sont nivelées derrière cette définition. Nous retrouvons ce genre de confusions dans quelques résumés de présentations faisant état $d^{\prime}$ «apprenants francophones», de «francophones vietnamiens » ayant des difficultés communicatives, ou encore de «traducteurs francophones au Vietnam» (SJDF, 2017). S'agit-il d'apprenants du FLE, de natifs (à défaut pour le moment d'une autre terminologie) ou de personnes ayant le français comme langue seconde? Pour éviter tout malentendu, les termes «Français », «Francophones» et «Francophiles» sont préférés dans une présentation (SJDF, 2017). Cependant, le terme Francophile fait débat au sein de la francophonie. Trop centré sur la 
France, il ne paraît pas adhérer à l'esprit de diversité. Il ne rend effectivement pas compte de l'intérêt envers les autres cultures francophones; un Francophile étant une personne qui « aime la France, les Français » (Francophile, 2015, p.519). Si la contextualisation trouve ici tout son sens (Chiss, 2017), rien ne devrait empêcher de distinguer un apprenant d'un autre. La catégorisation de Francophone est " pertinente et s'impose pour conserver une démarche scientifique pour avoir une vision réaliste et intelligible» (Observatoire de la langue française de l'OIF, 2014, p. 8). Francophone recouvre une multitude de personnes aux profils extrêmement variés qu'il est dommage d'occulter dans notre discipline. Les apprenants du français ou les natifs ne ressentent pas les mêmes besoins. Cette différenciation concernant les enseignants est aussi constructive, car les intérêts, les besoins pédagogiques, les méthodologies diffèrent selon les contextes et les personnalités. Le vécu, les sensibilités vis-à-vis des langues des enseignants ne sont pas à dissoudre sous le terme générique francophone. La définition de celui-ci ne devrait donc pas se situer dans le paradigme des aptitudes, mais dans celui des expériences vécues. A ce propos, les compétences langagières de nos collègues venus de pays non membres de l'OIF ne sont nullement à remettre en cause; et nous partageons la fascination d'une répondante pour le haut niveau de compétences en français de nos collègues non-natifs. La question de l'enquête concernant les gênes occasionnées par l'unique recours au français pendant le congrès illustre d'ailleurs très bien les compétences langagières de chacun : sur 111 répondants, seulement 2 personnes ont répondu «à peu près d'accord », c'est-àdire un peu gênées.

\section{Le français vous a gêné(e) par moment.}
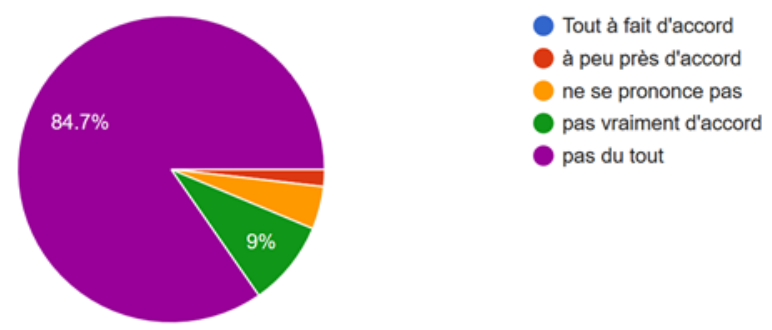

Figure 1. Réponses sur les gênes liées à l'emploi du français dans le congrès

L'une des raisons évoquées est le recours à l'argot. Rappelons que ce langage évolue constamment et peut effectivement constituer une gêne pour tous ceux à qui l'occasion de mettre à jour les connaissances lexicales argotiques manque. Ce phénomène peut aussi bien toucher un natif qu'un nonnatif. Il a aussi été apprécié par 96.5\% de 114 répondants que le français ait été l'unique langue officielle de CAP-Kyoto2017.

L'Observatoire de la langue française de l'OIF (2014, p. 8) en a conscience : « les francophones éprouvent des réalités francophones bien différentes ». Il en va de même dans l'enseignement du français et il paraît difficile de rendre compte et de véhiculer des idées de la diversité si tout est englobé dans un mot.

Chaudenson (2008, p. 23) évoquait dans ses travaux de 1989 les termes de «francophones", "francophonoides» et «franco-aphones". Pour notre part, nous nous appuyons sur l'Observatoire de la langue française de l'OIF (2014, p. 8) et ses trois planètes de locuteurs "naître en français », "vivre en français » et " choisir le français comme langue étrangère » pour définir comme Francophone tout natif ayant suivi une partie de sa scolarité obligatoire dans un pays francophone ou en français. Ainsi, nous retrouvons les Francophones du Canada, de France, de Tunisie, de SainteLucie, etc. Nous rejoignons ceux qui ont fait 
le choix d'apposer le suffixe «phile» provenant du grec «philos» (personne qui aime) à cette appellation pour qualifier toute personne ayant un intérêt pour le français. De ce fait, le terme Francophonophile débutant, avancé ou confirmé, justifiera des progrès ou du niveau de la personne concernée. Au regard des profils et des vécus aussi variés que surprenants, une personne peut être tantôt qualifiée de francophone ou de francophonophile selon les arguments mis en avant.

\subsection{Les langues à enseigner dans une perspective de la diversité des langues}

Le fait de reconnaître les Francophones des Francophonophiles permet aussi de réduire les confusions entourant les langues enseignées. Tant dans les approches que dans les contenus, les enseignements du Français Langue Maternelle (FLM), Français Langue Seconde (FLS), FLE ou autres, sont sensiblement différents. Les personnes présentes au congrès en charge de cours autres que ceux du FLE pourront particulièrement en témoigner.

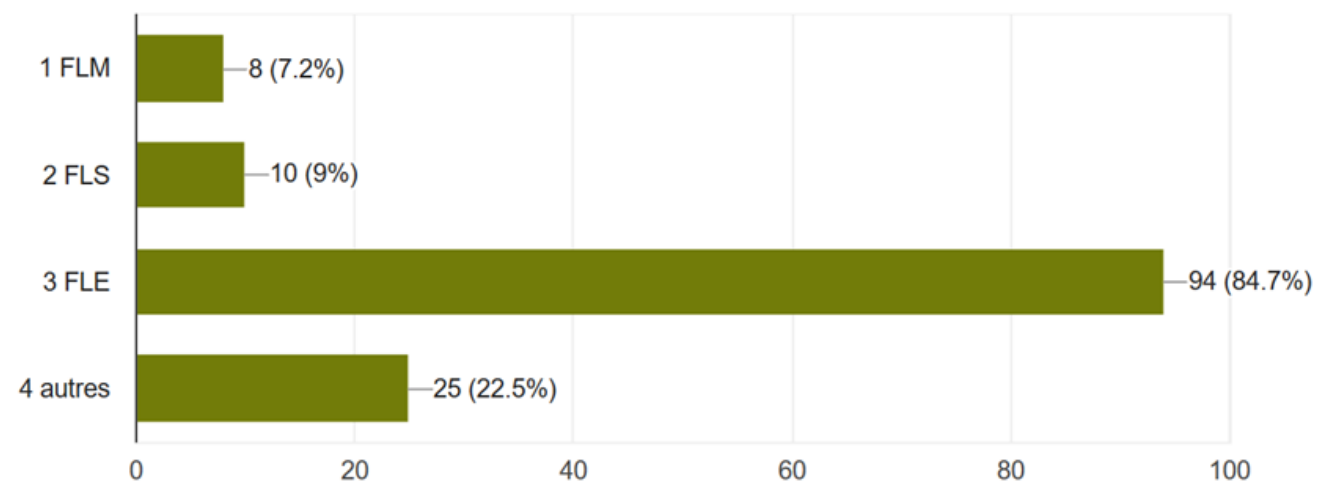

Figure 2. Langues enseignées par une partie des congressistes

Les travaux des spécialistes de la francophonie mettant l'accent sur la francophonie et ses univers nous offrent des opportunités de repenser les langues dans une perspective de la diversité des langues en didactique du français. Pour Riffard, dans Francophonie littéraire: quelques réflexions autour des discours critiques, (2006, cité dans Berthelot, 2015, p. 26-28) : «le raisonnement dans le domaine des études francophones se fait davantage en termes d'espace que d'histoire ». L'espace francophone est propice aux foisonnements des langues, offrant des environnements bilingues ou plurilingues. De par leur profession exercée, les congressistes sont très au fait de ces situations. De plus, ils sont en majorité bilingues, voire trilingues ou polyglottes comme l'indique le tableau ci-dessous :

Tableau 1. Langues utilisées indépendamment du français pendant le congrès

\begin{tabular}{|c|c|c|c|c|c|}
\hline Langues & $\begin{array}{l}\text { Nombre de } \\
\text { répondants }\end{array}$ & Langues & $\begin{array}{l}\text { Nombre de } \\
\text { répondants }\end{array}$ & Langues & $\begin{array}{l}\text { Nombre de } \\
\text { répondants }\end{array}$ \\
\hline français & 20 & & ? & 3 langues: & \\
\hline uniquement & 20 & russe & 2 & anglais/ arabe/ chinois/ & 5 \\
\hline anglais & 19 & arabe & 1 & hindi/ japonais/ russe & \\
\hline japonais & 10 & coréen & 1 & 4 langues et + : & \\
\hline vietnamien & 4 & hindi & 1 & $\begin{array}{l}\text { anglais/ chinois/ coréen/ } \\
\text { japonais/ vietnamien }\end{array}$ & 2 \\
\hline chinois & 3 & & & & \\
\hline
\end{tabular}

Les langues étrangères sont inclues dans la diversité linguistique, mais ne sont pas les seules langues usitées. Les langues francophones sont assurément présentes et vivaces. Comme tant d'autres, Bonn (2008) reconnaît, par exemple, la vigueur et la pluralité des langues francophones d'un point de vue littéraire. Ses propos cités ci- 
dessous ont l'avantage de s'étendre au domaine pédagogique :

La diversité des langues françaises au sein de la francophonie est bien plus grande en tout cas que la différence entre traductions en français standard de textes étrangers utilisés à l'agrégation de Lettres modernes. (...) La francophonie est d'abord une multiplicité de cultures auxquelles des langues diverses sont rattachées, et entre lesquelles des langues françaises diversifiées permettent un comparatisme plus réaliste et plus actuel que la frileuse clôture intra-européenne des programmes d'enseignements comparatistes en France. » (p. 44)

Les langues francophones sont constamment au contact de langues minoritaires, les premières concernées par l'extinction. Elles ont des parcours communs, s'entremêlent parfois. Les liens intimes qu'elles ont toutes su créer dans le temps peuvent être bénéfiques aux langues minoritaires. Les langues francophones ont le bagage suffisant pour faire vivre dans les cours de français les langues minoritaires qu'elles côtoient. La reconnaissance de la diversité linguistique en didactique du français passe alors par l'acceptation des langues francophones. Or, ces dernières sont absentes des propos de la plupart des répondants de l'enquête. La question portant sur les nouveaux mots et expressions entendus pendant le congrès décèle cette tendance. Sur 36 personnes, 25 affirment ne pas en avoir entendu; trois ne s'en souviennent plus; cinq personnes mentionnent des mots étrangers tels que «kanpai » ou «bento ». Des trois personnes restantes, une rapporte un vocabulaire argotique du français standard (« blé » faisant référence à l'argent), et deux font effectivement référence au répertoire de langues francophones avec les mots «nonante» et «présentement». Il est à déduire de ces résultats que le français standard a été privilégié dans les échanges. Son utilisation s'explique notamment par la volonté de se faire comprendre ou par la formation suivie. Sur 120 répondants des congressistes venus de 56 pays, dont 17 pays membres de l'OIF ou considérés francophones (Algérie et Israël), 56 personnes déclarent avoir effectué leur principale formation de professeur de français en France, et 17 personnes disent l'avoir passée dans un pays de l'OIF. Avec une majorité de répondants ayant acquis des connaissances didactiques dans l'hexagone, le français de France semble être la langue francophone de référence. Est-ce que les professeurs n'enseignent pas par la suite la langue à laquelle ils se sont formés? Le recours et la diffusion d'une langue standardisée ne facilitent pas la promotion de la diversité linguistique. Un français pluriel mérite d'exister en didactique du français pour défendre la diversité, et pourtant les langues francophones (l'acadjonne, le cadien, le franglais du Cameroun ou l'occitan) ne figurent pas dans le programme du congrès. Ne devrions-nous pas passer d'une didactique du français à une didactique des langues francophones pour assurer l'existence de la diversité linguistique? $\mathrm{Ne}$ devrions-nous pas concevoir des objectifs pédagogiques autour des langues francophones? S'il semble effectivement plus simple d'élaborer des curricula intégrant les langues francophones du Canada, de la Belgique ou de la Suisse, où de nombreuses universités partenaires de celles des pays exolingues accueillent des étudiants en échange, l'enseignement des langues francophones $\mathrm{d}^{\prime}$ Afrique se révèle plus complexe. Kasuya (2017) emprunte pourtant ce chemin avec une correspondance organisée entre des apprenants japonais et nigériens. Cette communication devait être intéressante en tout point et permettre d'appréhender les langues adoptées dans cette correspondance. S'agissait-il du français, d'une langue francophone du Niger? Le contexte ivoirien avec ces trois variétés de français (le français populaire ivoirien, le français ivoirien et le nouchi) est lui-aussi un cas d'enseignement intéressant. La croissance de l'enseignement du français comme spécialité dans les universités chinoises est spectaculaire et unique au monde avec une augmentation de 340\% entre 1999 et 2014 (Observatoire de la langue française de l'OIF, 2014, p. 290-310). Est-ce que les étudiants chinois de français, tournés vers l'Afrique pour des raisons économiques, ne devraient pas être aujourd'hui les 
apprenants des langues francophones? Ce sillon est à creuser; des formations en didactique des langues francophones et les compétences langagières appropriées s'imposent. Dans le domaine de la didactique des langues francophones, beaucoup reste à faire, comme en témoigne leur sousreprésentation dans les discours des intervenants. Si nous nous référons à la fréquence des termes employés (SJDF, 2017), nous comprenons que les intérêts didactiques n'étaient pas souvent orientés vers les thèmes de CAP-Kyoto2017. La diversité des langues a été finalement peu traitée. 57 interventions sur plus de 250, soit un cinquième, font mention de la francophonie (29) et/ou de la diversité (28) (L'erratum du 15/09/2017 est pris en compte dans ce calcul). Bien sûr, cela ne signifie pas que ces thèmes n'aient pas été abordés pendant les différentes interventions, et que lesdites interventions ne se rattachent pas aux axes de recherches proposés. Toutefois, ils n'ont pas été mis en avant. Les priorités didactiques des intervenants se sont situées ailleurs, pour la plupart. Les termes cultures et francophones sont associés 2 fois ("cultures francophones" et «comportements culturels francophones »), et ceux de langue et francophone ne sont associés qu'une fois sous la forme "politiques linguistiques francophones ». Aux propos des intervenants répondent ceux d'une partie des participants via l'enquête. Les termes évoquant la multiplicité du français se sont faits rares. Langue au pluriel se manifeste à neuf reprises, quand ceux de langues françaises et variétés $d u$ français sont constatés respectivement qu'une seule fois. Le terme de langues francophones n'est pas usité. Les réponses ne tiennent qu'en une ligne et le fond des pensées n'est pas ici exprimé. Cependant, les termes choisis par les répondants matérialisent les idées prioritaires.

Il est dit que la francophonie doit s'engager « à soutenir les autres langues et à prendre en charge leur développement à concevoir non comme une menace mais comme un enrichissement » (Grandguillaume, 2008, p. 55-56). L'enseignement du français tel qu'il l'est aujourd'hui ne remplit pas les conditions nécessaires pour impulser la diversité linguistique, bien qu'il ne soit pas incompatible avec la francophonie ou l'initiation d'une culture francophone. Il est courant de trouver des établissements supérieurs dispenser des cours de français et organiser la semaine de la langue française et de la francophonie. Effectivement, il est plus aisé d'enseigner une langue française standardisée, écrite, comprise par un grand nombre de locuteurs, qu'une langue francophone à l'évolution rapide (un créole par exemple), limitée à un espace, et donc comprise par une communauté précise.

\section{CONCLUSION}

Nous comprenons ici que la diversité des langues (FLE, langues étrangères, mais encore langues francophones) n'est pas si évidente à défendre quand la part belle est faite à une langue aux critères standardisés. Quelle que soit la région où il exerce, le corps professoral propage un modèle de français de par son discours et ses pratiques en vigueur dans la salle de cours, influe sur les représentations $\mathrm{du}$ français que les apprenants construisent. Ce phénomène se traduit différemment selon les contextes. A titre d'exemple, un français standard est dispensé dans les classes togolaises quand des variations grammaticales présentes dans les discours ne sont pas explicitées par les enseignants (Peuvergne, 2008, p. 95). Nous pouvons également prendre le cas du Japon où le français enseigné dans les départements de spécialité des universités est «muséifié par une vision patrimoniale persistante » amenant les étudiants essentiellement sur une voie littéraire (Observatoire de la langue française de 1'OIF, 2014, p. 254-255), celle de la littérature française. A l'instar d'un répondant de l'enquête, « adepte d'une approche variationniste du ou plutôt des français et même de toute langue ", nous pensons qu'encourager la multiplicité du français dans la classe fera vivre la diversité linguistique. Il est à noter que certaines décisions politiques compliquent la tâche. Comme l'a stipulé Del Pietro (2017) dans sa conférence inaugurale, la France, pays membre de la Francophonie, ne défend pas 
expressément la diversité des langues puisqu'elle a refusé de ratifier la Charte européenne des langues régionales ou minoritaires, signée en mai 1999 (Conseil de l'Europe, s. d.). Ce traité faisant de la protection et de la promotion des langues historiques régionales et des langues minoritaires en Europe une priorité, est en contradiction avec la constitution stipulant que « la langue de la République est le français » (franceinfo, 2015). Toutefois, ce refus de la France ne semble pas constituer un frein au dynamisme de la communauté scientifique. Le programme de recherche « Langue française, diversité culturelle et linguistique : culture d'enseignement et culture d'apprentissage (CECA) » se penche sur les comportements des enseignants et des apprenants dans la classe de langue de 20 pays afin de les comprendre (FIPF, s. d.). Il aurait été intéressant de retrouver ce projet (renouvelé) au congrès, avec ses données observables récoltées et ses savoir-faire scientifiques et pédagogiques mutualisés par des équipes de recherche éloignées géographiquement les unes des autres, pour répondre aux intérêts de certains congressistes voulant « acquérir d'autres méthodes et savoir ce qui se passe en dehors de la Jordanie », « comprendre la direction que prend la francophonie ", ou encore obtenir « une large vision sur l'enseignement du français ».

$\mathrm{Au}$ Japon, pays où s'est tenu le congrès (comme dans les autres pays exolingues selon nous), les enseignants de français doivent prendre conscience qu'ils sont l'un des rouages de cette francophonie (Komatsu, $2017 b$, p. 75), et que la didactique des langues francophones doit être placée au centre de la promotion de la diversité linguistique. Cette prise de conscience générale déclenchera des dispositifs d'enseignements amenant les apprenants à s'éveiller à la diversité des langues. Aux questions de l'enquête sur les points appréciés de CAP-Kyoto2017, sont revenues à de très nombreuses reprises la création de liens personnels, la découverte d'une francophonie en Asie, la découverte $d^{\prime}$ une langue n'appartenant pas seulement à la France. La conduite d'un tel congrès rassemblant les professeurs de français dans un pays exolingue a permis ainsi de communier un esprit de diversité dans une langue en partage et de ressentir cette francophonie à défaut $\mathrm{d}^{\prime} \mathrm{y}$ avoir, sur le moment, mis les mots.

\section{REMERCIEMENTS}

Nous tenons à remercier tous ceux qui ont pris le temps de répondre à cette faste enquête. Nous devons son existence au soutien de la FIPF, qui comme sponsor, s'est proposée de remettre un prix à deux répondants tirés au sort. Cette collaboration a valu un relatif succès de participation dépassant nos attentes. Enfin, nous remercions nos collègues du Comité d'Organisation de CAP-Kyoto2017, et plus particulièrement Leroux Brendan et Kuradate Ken-ichi pour leur contribution à l'élaboration de l'enquête.

\section{RÉFÉRENCES}

Awad, K. et Thibeault E.-N. (2017, septembre). Qu'est-ce que la Francophonie? Atelier dispensé au 4ème Congrès régional de la Commission Asie-Pacifique, Kyoto, Japon. Résumé repéré http://cap2017kyoto.sjdf.org/program me

Berthelot, R. (2015). Littératures francophones en classe de FLE. Pourquoi et comment les enseigner. Paris, France : L'Harmattan.

Bonn, C. (2008). Pour un comparatisme français ouvert à la francophonie et aux métissages culturels. Plaidoyer en forme de polémique. Dans K. Holster et I. Skattum (dir.), La francophonie aujourd'hui: Réflexions critiques (p. 4148). Paris, France : L'Harmattan.

Chaudenson, R. (2008). La Francophonie vue de Sirius. Dans K. Holster et I. Skattum (dir.), La francophonie aujourd'hui: Réflexions critiques (p. 11-26). Paris, France : L'Harmattan.

Chiss, J.-L. (2017, septembre). Le français: contextualisations et idéologies linguistiques. Conférence présentée au 4ème Congrès régional de la Commission Asie-Pacifique, Kyoto, Japon. Résumé repéré à 
http://cap2017kyoto.sjdf.org/program me

Conseil de l'Europe. (s.d.). Etat des signatures et ratifications du traité 148 . Repéré

https://www.coe.int/fr/web/convention s/full-list/-

/conventions/treaty/148/signatures

Del Pietro, J.-F. (2017, septembre). L'école suisse peut-elle contribuer à une écologie des langues. Conférence présentée au 4 ème Congrès régional de la Commission Asie-Pacifique, Kyoto, Japon. Résumé repéré à http://cap2017kyoto.sjdf.org/program me

Fédération Internationale des Professeurs de Français, (FIPF). (s. d.). Présentation du projet CECA. Repéré à http://fipf.org/recherchedidactique/ceca

Franceinfo. (2015). Le Sénat rejette la Charte des langues régionales: pourquoi la ratification coince encore et toujours?. Repéré https://www.francetvinfo.fr/elections/r egionales/charte-des-languesregionales-pourquoi-la-ratificationcoince-encore-ettoujours_1147447.html

Francophile. (2015). Dans I. Jeuge-Meynart (dir.), Le grand Larousse illustré 2015 (p. 519). Paris, France : Larousse.

Grandguillaume, G. (2008). La francophonie vue du monde arabe et du Maghreb. Dans K. Holster et I. Skattum (dir.), La francophonie aujourd'hui: Réflexions critiques (p. 49-60). Paris, France: L'Harmattan.

Kasuya, M. (2017, septembre). Échange culturel par correspondance avec le Niger. Communication présentée au 4ème Congrès régional de la Commission Asie-Pacifique, Kyoto, Japon. Résumé repéré http://cap2017kyoto.sjdf.org/program me

Klinkenberg, J.-M. (2015). La Langue dans la cité - Vivre et penser l'équité culturelle. Bruxelles, Belgique: Les Impressions Nouvelles.
Komatsu, S. (2017a). 国際フランス語教授連 合（FIPF）第 4 回アジア太平洋大会（ 京都大会）報告/ Compte rendu du 4 ème congrès régional de la Commission Asie-Pacifique (Congrès de Kyoto) de la Fédération Internationale des Professeurs de Français (FIPF). ふらんす, 12 月号/ furansu, numéro de février. Repéré à https://webfrance.hakusuisha.co.jp/pos ts $/ 223$

Komatsu, S. (2017b). フランス語、フランコ フォニーの今日の課題：Jean-Marie Klinkenberg の著書より考える/ Le français, les devoirs de la francophonie actuelle: Réflexions sur l'ouvrage de Jean-Marie Klinkenberg. Rencontres, 31, 72-75.

Observatoire de la langue française de l'Organisation Internationale de la Francophonie. (2014). La langue française dans le monde 2014. Paris, France : Nathan.

Peuvergne, J. (2008). Variation du groupe verbal et discours sur les langues: aperçu des représentations et pratiques d'enseignants togolais. Dans K. Holster et I. Skattum (dir.), La francophonie aujourd'hui: Réflexions critiques (p. 89-107). Paris, France: L'Harmattan.

Société Japonaise de Didactique du Français, (SJDF). (2017). Programme du 4ème Congrès régional de la Commission Asie-Pacifique : Ecologie du français \& Diversité des langues. Repéré à http://cap2017kyoto.sjdf.org/program me

Traisnel, C. (2015). La Francophonie, entre langue partagée et espace de négociation politique. La Revue Hermès, 71(1), 122-129. Repéré à https://www.cairn.info/revue-hermesla-revue-2015-1-page-122.htm 